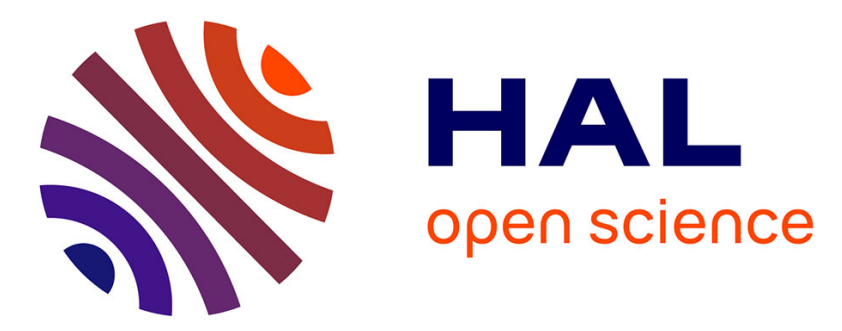

\title{
Automatic 3D segmentation of multiphoton images: a key step for the quantification of human skin
}

Etienne Decencière, Emmanuelle Tancrède-Bohin, Petr Dokládal, Serge

Koudoro, Ana-Maria Pena, Thérèse Baldeweck

\section{- To cite this version:}

Etienne Decencière, Emmanuelle Tancrède-Bohin, Petr Dokládal, Serge Koudoro, Ana-Maria Pena, et al. Automatic 3D segmentation of multiphoton images: a key step for the quantification of human skin. Skin Research and Technology, 2013, 19 (2), pp.115-124. 10.1111/srt.12019 . hal-00881692

\section{HAL Id: hal-00881692 \\ https:/ /hal-mines-paristech.archives-ouvertes.fr/hal-00881692}

Submitted on 7 Apr 2015

HAL is a multi-disciplinary open access archive for the deposit and dissemination of scientific research documents, whether they are published or not. The documents may come from teaching and research institutions in France or abroad, or from public or private research centers.
L'archive ouverte pluridisciplinaire HAL, est destinée au dépôt et à la diffusion de documents scientifiques de niveau recherche, publiés ou non, émanant des établissements d'enseignement et de recherche français ou étrangers, des laboratoires publics ou privés. 


\title{
Automatic 3D segmentation of multiphoton images: a key step for the quantification of human skin
}

\author{
E. Decencière ${ }^{\dagger}$, E. Tancrède-Bohin ${ }^{\star}$, P. Dokládal ${ }^{\dagger}$, S. Koudoro ${ }^{\dagger}$, \\ A.-M. Pena ${ }^{\star \star}$ and T. Baldeweck ${ }^{\star \star}$ \\ † Centre de Morphologie Mathématique, \\ Mathématiques et Systèmes, MINES ParisTech \\ * L'Oréal Research and Innovation, Centre de Recherche Bioclinique, \\ Hôpital Saint-Louis, Paris, France \\ ${ }^{\star \star}$ L'Oréal Research and Innovation, Aulnay-sous-Bois, France
}

October 23,2012

\begin{abstract}
Background/purpose: Multiphoton microscopy has emerged in the past decade as a useful non invasive imaging technique for in vivo human skin characterization. However, it has not been used until now in routine clinical practice, mainly because of the lack of specific image processing tools that would allow the investigator to extract pertinent quantitative three-dimensional (3D) information from the different skin components.

Methods: We propose a 3D automatic segmentation method of multiphoton images which is a key step for epidermis and dermis quantification. This method, based on the morphological watershed and graph cuts algorithms, takes into account the real shape of the skin surface and of the dermal-epidermal junction and allows separating in 3D the epidermis and the superficial dermis.

Results: The automatic segmentation method and the associated quantitative measurements have been developed and validated on a clinical database designed for aging characterization. The segmentation achieves its goals for epidermis-dermis separation and allows quantitative measurements inside the different skin compartments with sufficient relevance.
\end{abstract}


Conclusions: This study shows that multiphoton microscopy associated with specific image processing tools provides access to new quantitative measurements on the various skin components. The proposed 3D automatic segmentation method will contribute to build a powerful tool for characterizing human skin condition. To our knowledge, this is the first 3D approach to the segmentation and quantification of these original images.

Key words: Multiphoton microscopy, 3D image processing, segmentation, graph cuts, watershed, quantification, human skin

In the past decade, multiphoton microscopy has emerged as a useful non invasive technique for in vivo skin imaging, as it allows 3D data ( $\mathrm{x}-\mathrm{y}-$ z) on the epidermis and superficial dermis to be acquired with sub-cellular resolution. Multiphoton microscopy provides complement modalities, such as two-photon excited fluorescence (2PEF) and second harmonic generation (SHG), enabling non invasive imaging of skin to be performed through combined endogenous sources of contrast $[1,2]$. The specificity of these signals enables the various morphological structures of the skin to be identified. In epidermis, endogenous fluorophores (keratin, $\mathrm{NAD}(\mathrm{P}) \mathrm{H}$, flavins, melanin, ...) contribute to the $2 \mathrm{PEF}$ signal. In dermis, two specific signals are generated: on the one hand, the $2 \mathrm{PEF}$ signal is produced by elastin, yielding images of the elastic fiber network. On the other hand the SHG signal originates from fibrillar collagens, allowing collagen fiber networks to be visualized. Therefore, this microscopy technique provides a powerful tool for characterizing superficial parts of skin and their follow-up under various conditions.

Until now, multiphoton microscopy has not been used in routine clinical practice, mainly because of the lack of specific image processing tools that would allow the investigator to extract pertinent quantitative 3Dinformation from the different skin components. Indeed, a very few image processing methods have been developed for characterizing multiphoton images of human skin in vivo. In dermis, the proposed methods are mainly based on the computation of mean intensity of $2 \mathrm{PEF}$ and SHG signals, or of the density of pixels occupied by these signals on conveniently chosen $2 \mathrm{D}$ slices within dermis at a fixed depth from the skin surface [3, 4]. More recently, parameters linked to the morphology of keratinocytes in epidermis have been evaluated in $2 \mathrm{D}$ on several slices [5].

Assessing quantitative parameters in the $3 \mathrm{D}$ volume corresponding either to epidermis or to dermis requires to segment the multiphoton images in order to separate these compartments. Such a task is all but trivial, especially if the dermal-epidermal junction (DEJ) is neither flat nor parallel 
to the image plane. Up to our knowledge, no 3D approach to this issue has been previously proposed.

The goal of this work is to provide an automatic 3D segmentation method of multiphoton images of human skin, which is the key step for 3D quantification. This automatic 3D segmentation aims at separating the three main compartments appearing in the images: the coupling medium between skin and the cover glass (i.e. water), epidermis and dermis. Such a segmentation allows us to obtain a geometrical characterization of these compartments, as well as their interfaces, namely the skin surface and the dermal-epidermal junction. In addition, it opens the possibility to characterize the cells or the elastic and collagen fibers inside each 3D skin compartment in terms of quantity, size or organization.

\section{Multiphoton skin images}

\subsection{Clinical study}

The study involved 15 young (18-25 years) and 15 aged (70-75 years) human female volunteers. Before enrolment, each volunteer received information about the investigation procedure, was fully informed about the nature of the study and signed an informed consent. The experimental protocol was approved by the Saint-Louis Hospital (Paris) ethics committee and we followed the Declaration of Helsinki protocols. Multiphoton images were taken from the ventral side of the forearm.

\subsection{Multiphoton setup - DermaInspect $\AA$ device}

Multiphoton imaging was performed with the DermaInspect $\AA$ (JenLab GmbH, Jena, Germany), a CE-marked medical imaging system. SHG and 2PEF signals were simultaneously excited by a femtosecond Titanium-Sapphire laser adjusted to $760 \mathrm{~nm}$ as previously described [2].

The acquisition protocol entailed a trade-off between the microscope characteristics (wavelength, objective), the optical properties of the living tissue, the size of the structures to be characterized, and practical considerations (mainly acquisition time due to in vivo imaging). As a result, the optical resolution was very anisotropic. The sampling step being chosen in accordance with this resolution, the images also were highly anisotropic (the sampling step in the $z$ direction is 9 times larger than the sampling steps in the $x$ and $y$ directions). 
In practice, each acquisition produces two 3D images of $511 \times 511 \times 70$ voxels, with a sampling step of $0.255 \mu \mathrm{m}$ in the plane orthogonal to the optical axis $((x, y)$ plane), and of $2.346 \mu \mathrm{m}$ in the third direction ( $z$ axis). The first image corresponds to the $2 \mathrm{PEF}$ channel, and the second to the SHG channel. On each voxel, the grey level indicates the number of photons counted by the detectors.

\subsection{Image description}

The information contained in the 3D images is shown in Fig. 1. Top of the image (i.e. low $z$ values) corresponds to the coupling water medium. There, respective values of the $2 \mathrm{PEF}$ and SHG channels are very low $(<4$ photons), i.e. a noise range. Moving into the skin (increasing $z$ ) starts with the stratum corneum (Fig. 1a), composed of dead cells deprived of nuclei (corneocytes), and further the living epidermis (Fig. 1b-d), mainly containing keratinocytes. The lowest layer of epidermis is the basal layer (Fig. 1d), where keratinocytes divide before migrating upwards to the surface. This layer, also harboring melanocytes, typically shows the highest melanin density within keratinocytes. Logically these cells tend to generate a $2 \mathrm{PEF}$ signal stronger than the one produced by non melanin-enriched keratinocytes. Finally, going deeper through the DEJ interface, dermis is reached (Fig. 1e-f), where the $2 \mathrm{PEF}$ and SHG signals mainly originate from the elastic and collagen fibers.

\section{Automatic segmentation method}

The aim of the segmentation method is to classify each image voxel in one of the following compartments: coupling medium (CM), epidermis (ED) or dermis (D). The result will be a partition of the image domain into these three regions. Moreover, given that a typical clinical study based on these acquisitions may contain several hundred images, the segmentation method should not be time-consuming.

From a methodological image processing viewpoint, some characteristics of the multiphoton images have to be taken into account in the $3 \mathrm{D}$ segmentation method:

- The signal tends to be more intense near the central vertical axis of the image (i.e. the optical axis), and lower near the vertical borders.

- The signal intensity decreases with the imaging depth due to the attenuation and diffusion of excitation and emission light by the skin. 


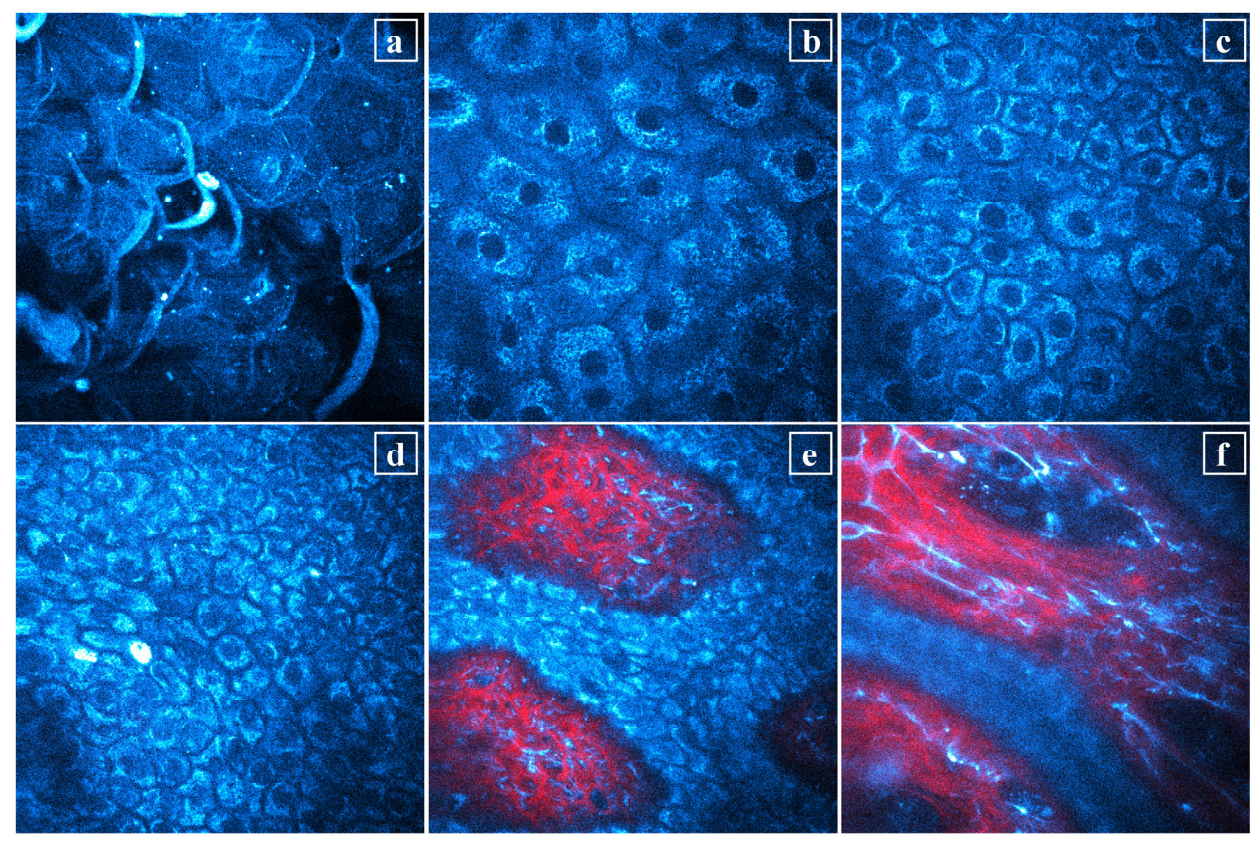

Figure 1: In vivo multiphoton images of normal human skin. 2PEF signal (cyan hot color) reveals the endogenous fluorophores distribution inside (a) corneocytes in the stratum corneum, keratinocytes in the upper (b) and lower (c) living epidermis, (d) keratinocytes in the stratum basale. In the dermis the $2 \mathrm{PEF}$ signal reveals elastic fibers whereas the SHG signal (red color) reveals the collagen fiber distribution: near DEJ fibers are clearly visible (e), whereas they become fuzzier with depth (f). Acquisition conditions: $40 \times, 1.3-\mathrm{NA}$ oil immersion objective; excitation power exponentially increased from $12 \mathrm{~mW}$ at the surface of the skin up to $47 \mathrm{~mW}$ at a depth of $75 \mu \mathrm{m}$ and kept constant for imaging depths greater than $75 \mu \mathrm{m}$; acquisition time: 7.4 seconds per image of $511 \times 511$ pixels and 8.5 minutes per stack of $130 \times 130 \mu \mathrm{m}^{2} \times 70$ images acquired every $2.346 \mu \mathrm{m}$. 
- The vertical resolution and sampling steps are large with respect to some structures: for example, at the top of the living epidermis, cells are horizontal and flat, their thickness being possibly smaller than the vertical resolution.

- The sampling steps in the $\mathrm{x}-\mathrm{y}-\mathrm{z}$ directions are not isotropic: large sampling step in the $z$ direction with respect to the sampling steps in the $x$ and $y$ directions.

- As the SHG signal is created by collagen fibers, one can use this information to identify the dermis. However, it is noteworthy that SHG signal alone does not allow the dermis to be delimited: at the DEJ level, dermal regions can be found with elastic fibers only and no collagen fibers, i.e. an absence of SHG signal.

All these features obviously make segmentation difficult. Based on the previous observations, we make the following assumptions the segmentation method will be based upon:

- The coupling medium (CM region) should be on the top of the image, and include the whole first slice. In this region, both $2 \mathrm{PEF}$ and SHG signals are low.

- The frontier between CM and ED regions, which should correspond to the skin surface, is characterized by a high gradient in the $2 \mathrm{PEF}$ signal. It tends to be horizontal.

- The upper part of epidemis (ED region), which should correspond to the stratum corneum, is indicated by high intensity $2 \mathrm{PEF}$ signal values, mainly generated by keratin in the corneocytes. In our experimental conditions, the mean $2 \mathrm{PEF}$ signal decreases when penetrating into the living epidermis, then increases again when coming near the DEJ. Indeed, the bottom layer of the epidermis, i.e. the basal layer, is often characterized by a high intensity $2 \mathrm{PEF}$ signal, at least near the optical axis.

- The upper part of dermis (D region) should theoretically show a significant SHG signal. However, as said above, collagen content may be highly variable among dermal areas near DEJ. On the other hand, $2 \mathrm{PEF}$ signal can be high in dermis when solar elastosis is present. Moreover, the junction between $\mathrm{D}$ and ED regions, corresponding to the DEJ, should be relatively smooth. 
- Finally CM region should not be in contact with D region. The three regions can be seen as three layers, the one corresponding to the epidermis separating those corresponding to the coupling medium and the dermis.

The segmentation method will have to enforce these hypotheses.

\subsection{Segmentation method overview}

The segmentation strategy was designed to overcome the above mentioned difficulties.

A marker-based approach [6] has been chosen. Markers are sets of pixels considered as specific to a given compartment. They are afterwards propagated to the rest of the image domain, in order to have a complete partition. A marker-based approach is appropriate for our case, as the a priori information we have allows us to build them with a good level of confidence.

The propagation is done in two steps. Firstly, a fast watershed algorithm produces a low-level segmentation, made of small, homogeneous regions, also called super-voxels. Secondly, the super-voxels are merged, taking into account the markers, thanks to a graph cut algorithm.

The general strategy is summed up by the flow chart shown in Fig. 2. All processing is done in $3 \mathrm{D}$, except otherwise specified.

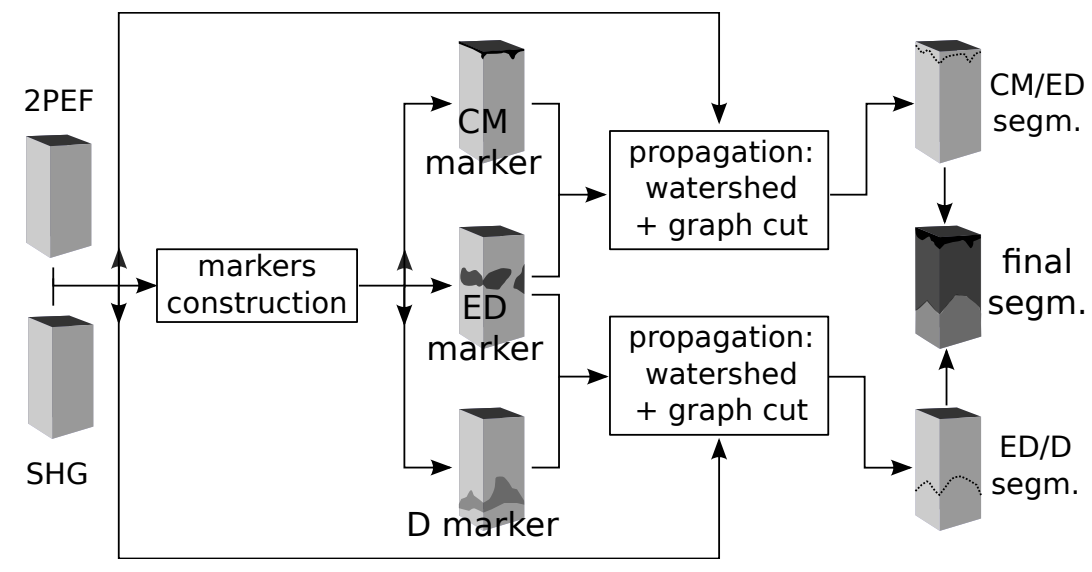

Figure 2: Flow chart of the 3D automatic segmentation method. 


\subsection{Markers construction}

The markers construction is based on thresholds of $2 \mathrm{PEF}$ and SHG signals. The thresholds used to compute the coupling medium and epidermis markers are obtained by dividing the maximal value of the $2 \mathrm{PEF}$ histogram by empirically chosen constants. Similarly, the threshold used to compute the dermis marker is obtained by dividing the maximal value of the SHG histogram by a constant. The threshold is applied to the corresponding channel (low $2 \mathrm{PEF}$ values for the coupling medium; high $2 \mathrm{PEF}$ values for epidermis; high SHG values for dermis).

Conflicts (i.e. a non empty intersection) between markers of the coupling medium and epidermis do not occur, since the corresponding thresholds are mutually exclusive. Conflicts between epidermis and dermis markers may occur, since high $2 \mathrm{PEF}$ values are generated both in epidermis (melanin,..) and in dermis (elastin). In such a case, priority is given to the dermis marker, since the SHG signal theoretically only originates from dermis.

The connected components of the resulting images touching a reference plane are kept: the plane $z=0$ for the coupling medium; $z=15$ for the epidermis; and the plane with the highest mean SHG value for the dermis.

\subsection{Markers propagation}

Several methods have been tested to propagate the markers. The watershed algorithm based on markers appears very efficient, albeit segmentation errors frequently appear near the image vertical borders, given the lack of a significant gradient in those regions [7]. In fact, we gave privilege to a method which uses gradient information when available, and otherwise minimizes the frontier area. Energy minimization methods offer a good approach to the problem. Graph cuts belong to this category [8]. A graph node is associated with each image voxel. When two voxels are neighboring, the corresponding nodes are linked with a graph edge. Two source nodes, traditionally called $s$ and $t$, are added to the graph, and linked to the nodes which correspond to marked voxels. A weight is associated with each edge. The resulting weighted graph is finally partitioned into two sub-graphs, the first of which contains $s$, and the second $t$. This is done thanks to a flow minimization algorithm. The method is such that the sum of the weights of the removed edges is minimal.

Graph cuts have been applied to large images, with three dimensions or more [9]. However, their theoretical complexity and memory requirements remain a problem in the present case, since several hundred 3D images have 
to be processed in a few hours. Therefore, instead of associating one node with each image voxel, we first compute a low level segmentation using the watershed approach, associate a node with each region of this low level segmentation, and add an edge between nodes such that the corresponding regions are neighbours. This approach was first proposed by Li et al. for $2 \mathrm{D}$ images [10], and further developed and applied to 3D, single-modality images by Stawiaski and Decencière [11]. Here we extend it to the multi-modality case. This approach affords the possibility of computing edge weights which take more information into account.

This kind of graph cut can only segment a graph into two sub-graphs, despite the goal of segmenting the 3D image into three regions. Given such a layered model, two steps were carried out: the frontier between the coupling medium and the epidermis was computed first, starting from their respective markers and second, analogously, the frontier between epidermis and dermis was computed.

In practice, starting from the morphological gradient of the supremum between the 2PEF and SHG channels, the low level segmentation is computed in $2 \mathrm{D}$, using a 4-connectivity, owing to the high anisotropy in the sampling steps (9 times larger in the $z$ direction). The objective of the graph cuts can therefore be seen as the fusion of the regions of the low level segmentation. The last choice to be made is that of the weights of the graph edges, which is an essential aspect of the design of graph cuts [12]. Several functions were tested. Let us consider two neighboring regions $A$ and $B$ of the low level segmentation. $N(A, B)$ denotes all the pairs of voxels $(p, q)$ such that $p$ and $q$ belong to $A$ and $B$ respectively, and $p$ and $q$ are neighbors (a 3D 6-connectivity is considered). The weight $w$ associated with the edge $e$ linking the corresponding nodes is:

$$
w(e)=\sum_{(p, q) \in N(A, B)} \frac{a(p, q)}{1+L_{1}(I(p), I(q))},
$$

where:

- $I(p)$ is the image value of voxel $p$, i.e. a couple $\left(I_{2 P E F}(p), I_{S H G}(p)\right)$ corresponding to the two channels measured on that point,

- $L_{1}(I(p), I(q))$ is the $L_{1}$ distance between the vector values $I(p)$ and $I(q)$, and

- $a(p, q)$ is the area between voxels $p$ and $q$. 


\subsection{Segmentation result}

The final result of the segmentation is a 3D image containing three gray levels, corresponding to the coupling medium (dark gray in the illustrations), epidermis (medium gray) and dermis (light gray), respectively. As an example, Fig. 3 illustrates the result of the application of this 3D automatic segmentation method to two different cases: a young and an old volunteers. The decrease in epidermis thickness and flattening of the dermal-epidermal junction with age are clearly evidenced. Fig. 4 shows the corresponding surface rendering of DEJ, whose flattening is clearly visible on these 3D reconstructions.

These qualitative observations based on the segmentation could also be made on the raw data, however the segmentation opens the possibility of computing quantitative measurements, on the segmentation frontiers, and on each layer.

\section{Quantification based on the 3D segmentation}

Our automatic 3D segmentation of multiphoton skin images opens the possibility to characterize inside each 3D skin compartment the cells or the elastic and collagen fibers, in terms of quantity, size or organization.

Several measurements, based on this segmentation, have been developed and tested. Here we describe those that have been used to validate the automatic 3D segmentation method (see section 4) on the one hand, and to characterize aging (section 5), on the other hand. They are classified into three categories: morphological, density and organization measurements.

\subsection{Morphological measurements}

Morphological measurements are directly derived from the segmentation, without further need of the original signals. They characterize the regions and frontiers shapes. Here, we only consider two of them.

The epidermis thickness, $T(E D)$, is the mean thickness, in $\mu m$, of the epidermis. The thickness of the epidermal compartment is measured along the $z$ axis at each $(x, y)$ position, and the mean value is computed.

The normalized area of DEJ is a generalization of the index of interdigitation described in two dimensions by Timár et al. on histological sections of human skin [13]. Let $A(D E J)$ be the area of this surface, and $A(H)$ the area of its projection on a horizontal plane. Then the normalized area is 

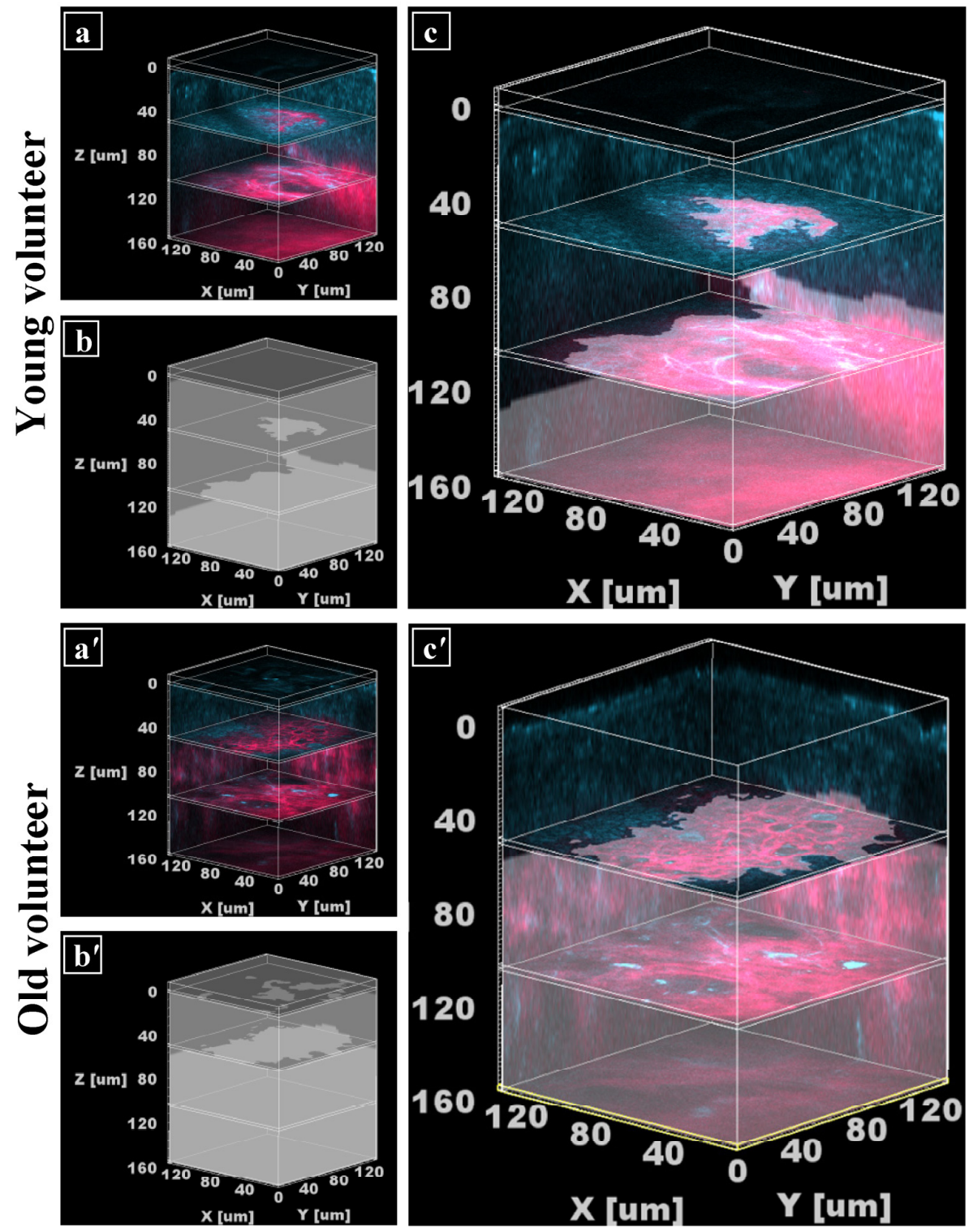

Figure 3: 3D results of the automatic segmentation applied to multiphoton images from a young (top) and an old (bottom) volunteers: (a) and (a') raw 2PEF/SHG data; (b) and (b') 3D automatic segmentation (coupling medium - dark gray, epidermis - medium gray and dermis - light gray); (c) and (c') overlay of 3D automatic segmentation on raw data. 


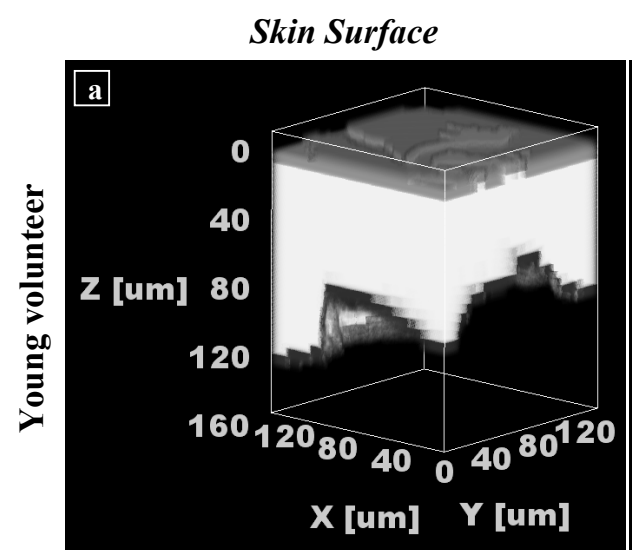

Dermal-epidermal junction (DEJ)
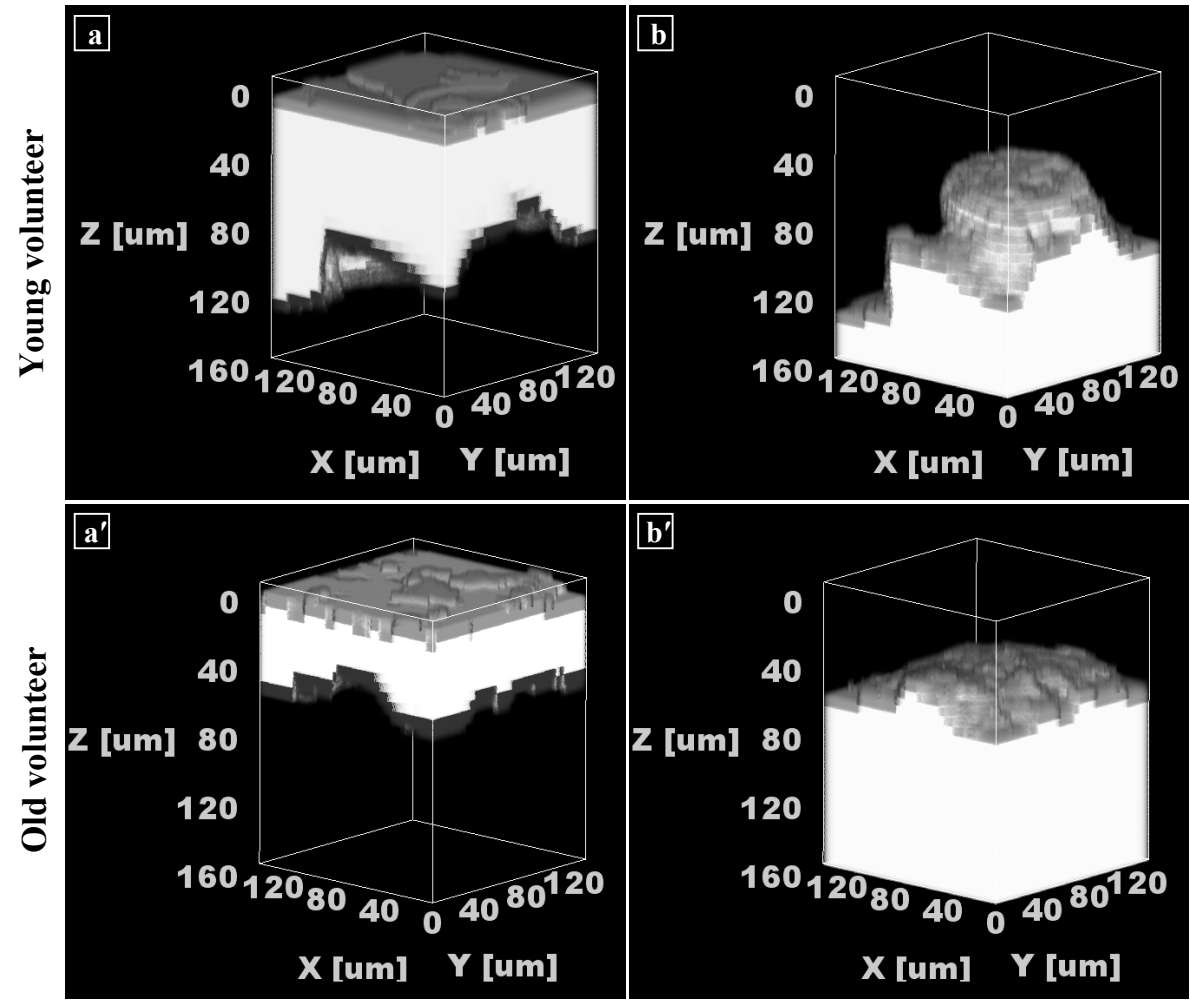

Figure 4: Surface rendering of the skin surface ((a) and (a')) and of DEJ ((b) and (b')), obtained using the automatic segmentation of multiphoton images from a young (top) and an old (bottom) volunteers. The flattening of DEJ with age is clearly visible. 
$N A(D E J)=A(D E J) / A(H)$. If its value is equal to 1 , then the DEJ is flat. The more the DEJ is undulated, the larger the parameter value.

\subsection{Density measurements}

$\operatorname{Sign}_{2 P E F}(D)$ and $\operatorname{Sign}_{S H G}(D)$ give the proportion of significant pixels in the dermis, for the $2 \mathrm{PEF}$ and SHG images, respectively. A pixel is considered as significant if its value is higher than a given threshold, here equal to 5 photons, for both channels. This value is such that, given the noise present in the images, a signal value higher than this threshold almost surely corresponds to a pixel where the signal is not only the result of noise. These measurements are used to characterize the density of elastic and collagen fibers.

We also estimate in $3 \mathrm{D}$ a quantitative parameter characterizing the dermis, the SHG-to-AF aging index of dermis $(S A A I D)$, which has been calculated in $2 \mathrm{D}$ in the literature $[3,4] .3 D-S A A I D$ of the dermis is a straightforward generalization of $2 D-S A A I D$ :

$$
3 D-S A A I D(D)=\frac{\operatorname{Sign}_{S H G}(D)-\operatorname{Sign}_{2 P E F}(D)}{\operatorname{Sign}_{S H G}(D)+\operatorname{Sign}_{2 P E F}(D)} .
$$

$3 D$-SAAID $(D)$ is between -1 and 1 . Negative values mean that there are more significant pixels with $2 \mathrm{PEF}$ than with SHG signals, and a value close to zero means that both proportions are similar. However, this value says nothing about their relative positions: do they coincide in the same pixels or not? In the following section, we propose a new measurement for this purpose.

\subsection{New multimodal organization measurement}

Multimodal organization measurements aim at quantifying the relative position of $2 \mathrm{PEF}$ and SHG signals. In order to measure the coincidence of $2 \mathrm{PEF}$ and SHG, we propose to measure the normalized overlapping $N O L(D)$ of both signals in the dermis:

$$
N O L(D)=\frac{\iiint_{D} 2 P E F_{n} \wedge S H G_{n}}{\iiint_{D} 2 P E F_{n} \bigvee S H G_{n}}
$$

where $\Lambda$ and $\bigvee$ are the infimum and supremum operators, respectively, and $2 P E F_{n}$ and $S H G_{n}$ correspond to the 2 PEF and SHG signals, normalized on the dermis so that their integrals are equal, i.e.: $\iiint_{D} 2 P E F_{n}=$ $\iiint_{D} S H G_{n} . N O L(D)$ is between 0 and 1; a large value means that the 
signals are spatially correlated; a low value indicates that they tend to be mutually exclusive. To our knowledge, this is the first time that this measurement is applied to multiphoton human skin images.

\section{Validation of the segmentation method}

Validating an automatic 3D segmentation method on complex biological data is a difficult task. The most straightforward approach is to ask at least two experts to produce a reference segmentation on a representative number of images.

\subsection{Reference segmentation}

Providing a reference segmentation on a representative number of images is an extremely tedious task. Therefore, we choose to build for each 3D multiphoton image of the data base a reference segmentation obtained by manually correcting the automatic segmentation on the whole data base. For that purpose, two experts inspected all the automatic segmentations, and manually corrected those which were not totally satisfactory. The resulting segmentations are hereafter called, for simplicity, the manual segmentations. Finally, measurements were recomputed using the manual segmentations.

\subsection{Performance evaluation}

One has to keep in mind that segmentation is only an intermediate step. The final goal of our clinical studies is to obtain an automatic segmentation, not necessarily pixel-to-pixel correlated to the manual one, but leading to a relevant estimation of the different quantitative measurements. Therefore, the issue is: are the measurements obtained thanks to these automatic segmentations accurate enough? For answering this question, the measurements described in the previous section were applied to the result of the automatic segmentation. If the proposed segmentation method is good enough for the current application, then the data obtained on the automatic and manual segmentations should be correlated, so that the same conclusions can be drawn from both sets of measurements.

Fig. 5 shows the correlation graphs for the six described measurements. It appears that the correlation does seem good. Indeed correlation coefficients are systematically above 0.84 . Correlation coefficients of morphological measurements, i.e. epidermis thickness $(T(E D))$ and normalized area of DEJ $(N A(D E J))$ are equal to 0.88 and 0.84 , respectively; they are smaller 
than those of the four other measurements within the dermis, which are higher than 0.93. As expected, the quality of segmentation has more impact on the measurements linked to the shape of the frontiers delimiting the epidermis (i.e. $T(E D)$ and $N A(D E J)$ ), than on those linked to the content of the skin compartments. Accordingly, one should be careful when using this automatic segmentation method in studies where a slight shape alteration of DEJ is to be characterized. In this case, a manual correction of the segmentation will be necessary.

Concerning the linear regression (as displayed on Fig. 5), the equation of the linear regression for each measurement leads to a slope coefficient close to 1 , and to an ordinate at origin close to 0 , which is a sign of non-bias.

In conclusion, we can state that our automatic segmentation achieves its goals and allows quantitative measurements inside the different skin compartments with sufficient relevance. Moreover, the current version of the segmentation method, which has not undergone any specific computational optimization, takes an average of one minute to process a 3D multiphoton image on a classical personal computer (Intel core i7, 8 GB memory).

\section{Application to aging characterization}

The clinical study (see section 1) has been designed to evidence age-related (young/old) differences. The aim of the study was to evaluate the relevance and interest of the 3D measurements obtained with the automatic segmentation method. Resulting data is given in Fig. 6 .

Morphological measurements For the mean thickness of epidermis, $T(E D)$, a decrease with age is noticed: mean value in the young group is $66 \mu \mathrm{m}$, whilst it decreases to $59 \mu \mathrm{m}$ in the old group.

Similarly, the normalized area of DEJ, $N A(D E J)$, which is related to the shape of DEJ, decreases with age (2.40 in the young group; 2.05 in the old group).

These age-dependent morphological changes are expected: with age, the epidermis becomes thinner and the rete ridges of the dermal-epidermal junction flatten out. It should be noted that, given the relatively small field of view, it was not evident that the measurements would reveal such morphological changes. Our 3D results are in agreement with the histology and other imaging techniques (optical coherence tomography, ultrasound imaging) performed on the same volunteers (data not shown) as well as with 

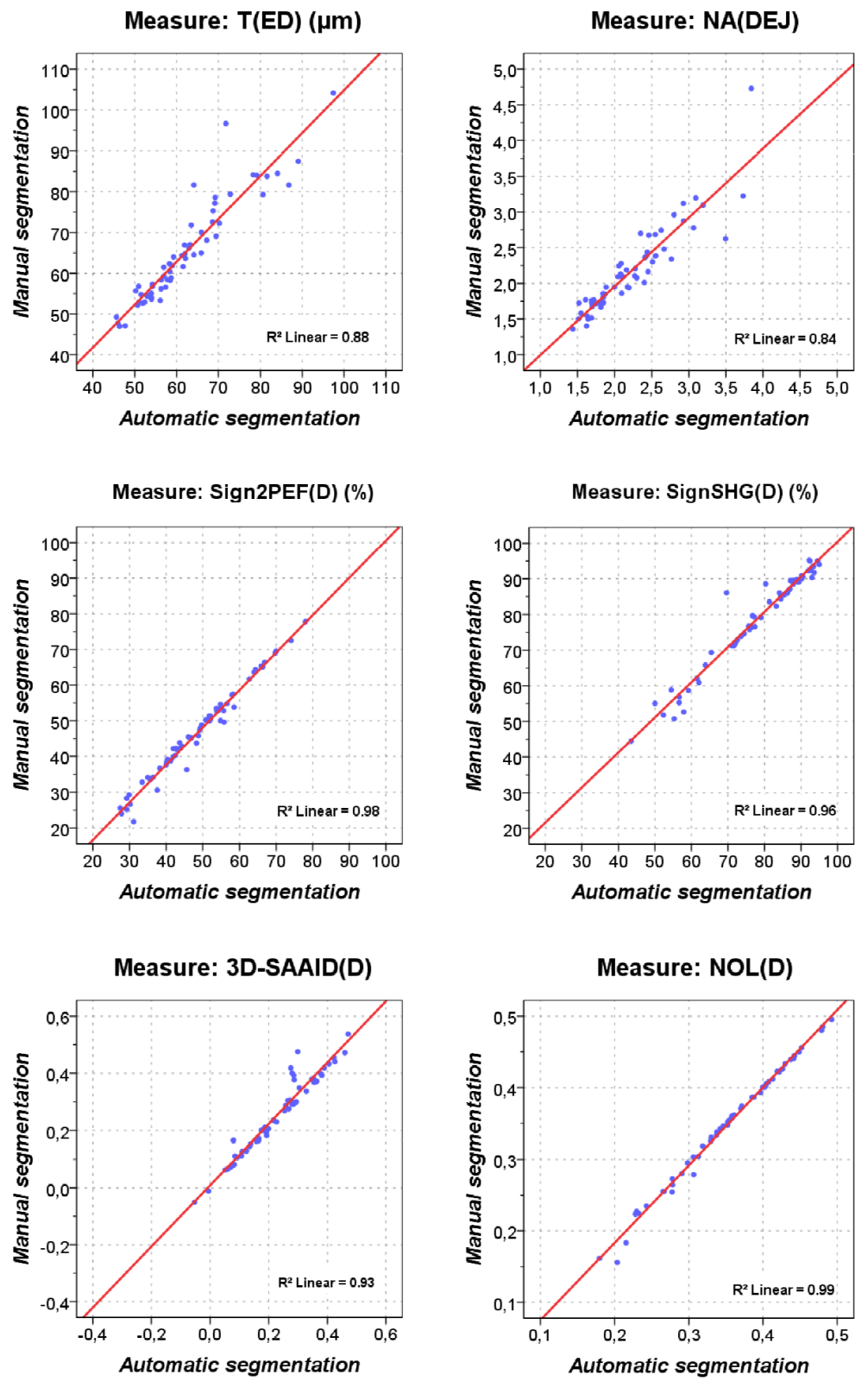

Figure 5: Correlation graphs for the 6 measurements: $T(E D), N A(D E J)$, $\operatorname{Sign}_{2 P E F}(D), \operatorname{Sign}_{S H G}(D), 3 D-S A A I D(D)$ and $N O L(D)$. Horizontal axis: values obtained with automatic segmentation; vertical axis: values obtained with manual segmentation. Each blue point represents the result obtained for one 3D multiphoton image; the linear regression fit is shown with a red line. 
published 2D data obtained with histology [14] or with other in vivo imaging techniques (optical coherence tomography, confocal microscopy) $[15,16]$.

Density measurements As regards $\operatorname{Sign}_{2 P E F}$ value, which is linked to the density of elastic fibers, an increase with aging is found. The results are in agreement with the histology performed on the same volunteers (data not shown). Sign ${ }_{S H G}$ value, which is linked to the density of collagen fibers, shows no difference between old and young volunteers.

We have also found that $3 D-S A A I D$ highly decreases with aging, as previously shown in $2 \mathrm{D}[17,3,4,18]$. This difference is directly linked to the relative increase in significant $2 \mathrm{PEF}$ pixels versus significant $\mathrm{SHG}$ pixels.

New organization measurement Lastly, the normalized overlapping $N O L(D)$ in the dermis tends to increase with age. This means that the correlation between $2 \mathrm{PEF}$ and $\mathrm{SHG}$ signals increases with age, which might correspond to a more disordered structure. This new parameter might be a new quantitative descriptor of the modifications of the superficial dermis organization during aging.

All the measurements have been made on the whole $3 \mathrm{D}$ region, either corresponding to epidermis or dermis in the 3D image. In our opinion, this is a clear advantage with respect to what has been previously reported in the literature for the dermis, i.e. taking empirically a $2 \mathrm{D}$ slice which is completely included within the dermis $[3,19,4]$. Not only the present approach avoids the selection of a $2 \mathrm{D}$ plane, but the measurements are based on a larger, more representative, volume of tissue.

In addition, all the data from automatic segmentation is consistent with data based on the manually corrected segmentation, which confirms that our automatic segmentation method is suitable for reliable quantitative characterization of human skin from multiphoton images.

\section{Conclusion and perspectives}

A 3D automatic segmentation method for analyzing in vivo multiphoton images of human skin has been presented. To our knowledge, this is the first 3D approach to the quantification of these images. Our method takes into account the real shape of the dermal-epidermal junction and is able to deal both with anisotropic sampling steps and spatially varying signal to

noise ratio. Existing and new measurements have been applied in 3D inside 

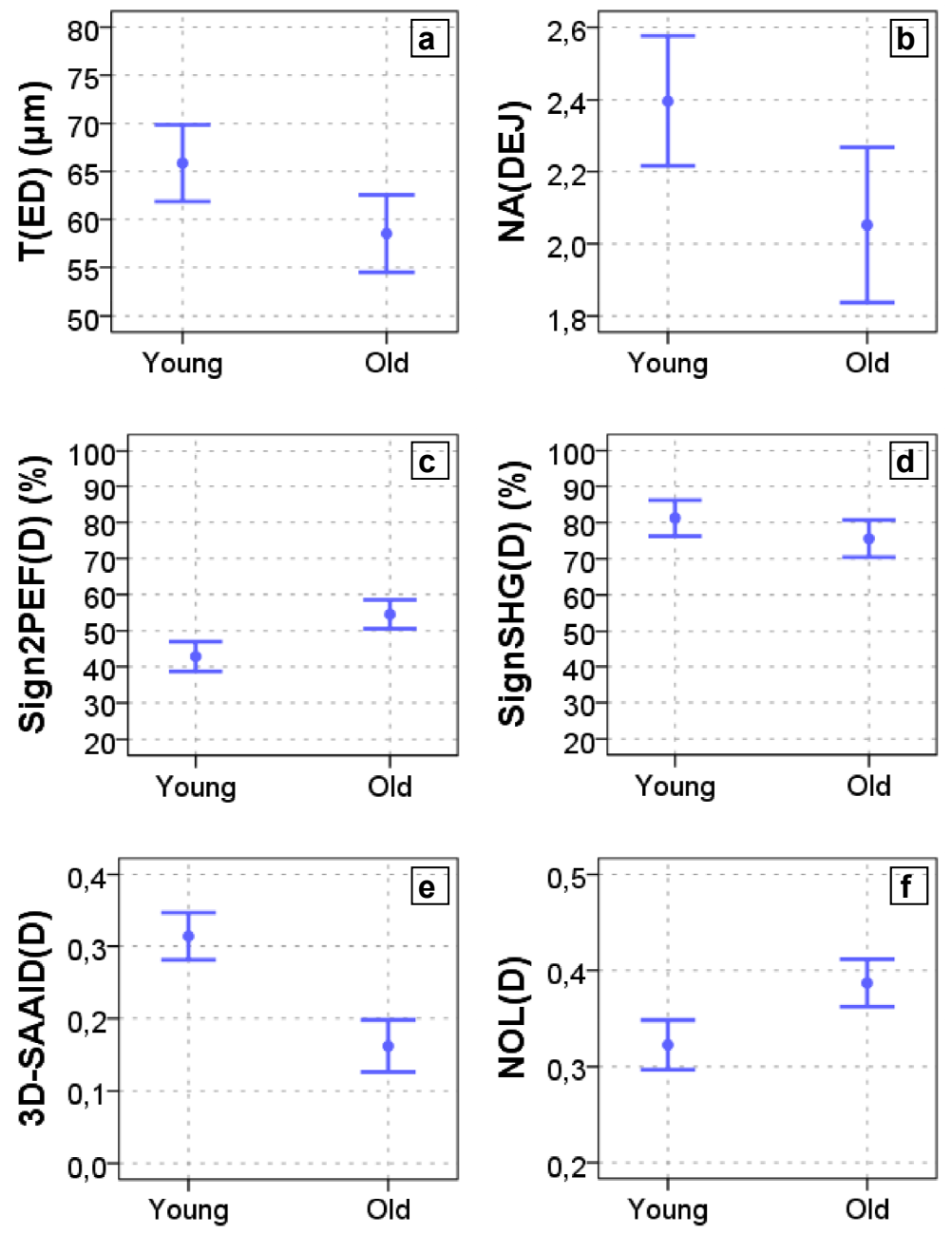

Figure 6: Quantification results obtained on the aging database: change with age in the mean thickness of epidermis (a), the normalized area of DEJ (b), the density of elastic (c) and collagen (d) fibers, the SHG-to-AF aging index of dermis (e) and the normalized overlapping of $2 \mathrm{PEF}$ and $\mathrm{SHG}$ signals in the dermis (f). Error bars correspond to $95 \%$ confidence intervals of the mean. 
each segmentation region. The automatic segmentation method and the associated quantitative measurements have been developed and validated on a clinical database designed for aging characterization. It is shown that the segmentation achieves its goals, and that the obtained measures are in agreement with previously reported results.

Segmentation is a key step prior to 3D quantification of the structures within each compartment, like epidermal cells and dermal fibers. This work has prompted several ongoing studies, such as the correction of the multiphoton signal attenuation, the development of new parameters for epidermis and dermis characterization, and the evaluation of the effects of different cosmetic and medical products on the skin using this method.

This study shows that multiphoton microscopy associated with specific image processing tools provides access to new relevant quantitative measurements on the various skin components. The development of this multidisciplinary approach associating multiphoton microscopy and image processing tools will be essential for the future deployment of this imaging technique in routine clinical practice.

\section{References}

[1] T. Baldeweck, E. Tancrede-Bohin, P. Dokladal, S. Koudoro, V. Morard, F. Meyer, E. Decenciere, and A. Pena, "In vivo multiphoton microscopy associated to 3D image processing for human skin characterization," in "Proc. SPIE 8226," (2012).

[2] H. Ait El Madani, E. Tancrede-Bohin, A. Bensussan, A. Colonna, A. Dupuy, M. Bagot, and A. Pena, "In vivo multiphoton imaging of human skin: assessment of topical corticosteroid-induced epidermis atrophy and depigmentation," Journal of Biomedical Optics 17 (2012).

[3] M. J. Koehler, K. König, P. Elsner, R. Bückle, and M. Kaatz, "In vivo assessment of human skin aging by multiphoton laser scanning tomography," Optics Letters 31, 2879-2881 (2006).

[4] K. Sugata, O. Avaro, T. Sano, and Y. Takema, "Evaluation of photoaging in facial skin by multiphoton laser scanning microscopy." Skin Research and Technology 17, 1-3 (2011).

[5] M. J. Koehler, S. Zimmermann, S. Springer, P. Elsner, K. König, and M. Kaatz, "Keratinocyte morphology of human skin evaluated by in 
vivo multiphoton laser tomography," Skin Research and Technology 17, 479-486 (2011).

[6] F. Meyer and S. Beucher, "Morphological segmentation," Journal of Visual Communication and Image Representation 1, 21-46 (1990).

[7] S. Beucher and C. Lantuéjoul, "Use of watersheds in contour detection," in "International workshop on image processing, real-time edge and motion detection," (1979).

[8] Y. Boykov, O. Veksler, and R. Zabih, "Fast approximate energy minimization via graph cuts," in "Proceedings of the International Conference on Computer Vision (ICCV)," , vol. 1 (1999), vol. 1, p. 377-384.

[9] Y. Boykov and G. Funka-Lea, "Graph cuts and efficient N-D segmentation," International Journal of Computer Vision 70, 109-131 (2006).

[10] Y. Li, J. Sun, C. Tang, and H. Shum, "Lazy snapping," ACM Trans. Graph. 23, 303-308 (2004).

[11] J. Stawiaski and E. Decencière, "Region merging via graph cuts," Image Analysis and Stereology 27, 39-46 (2008).

[12] L. Grady and M. Jolly, "Weights and topology: A study of the effects of graph construction on 3D image segmentation," in "Medical Image Computing and Computer-Assisted Intervention - MICCAI 2008," , vol. 5241 of Lecture Notes in Computer Science, D. Metaxas, L. Axel, G. Fichtinger, and G. Székely, eds. (Springer Berlin / Heidelberg, 2008), pp. 153-161.

[13] F. Timár, G. Soós, B. Szende, and A. Horváth, "Interdigitation index - a parameter for differentiating between young and older skin specimens," Skin Research and Technology 6, 17-20 (2000).

[14] R. Lavker, P. Zheng, and G. Dong, "Aged skin: a study by light, transmission electron, and scanning electron microscopy," The Journal of Investigative Dermatology 88, 44s-51s (1987).

[15] K. Sauermann, S. Clemann, S. Jaspers, T. Gambichler, P. Altmeyer, K. Hoffmann, and J. Ennen, "Age related changes of human skin investigated with histometric measurements by confocal laser scanning microscopy in vivo," Skin Research and Technology 8, 52-56 (2002). 
[16] B. Querleux, T. Baldeweck, S. Diridollou, J. de Rigal, E. Huguet, F. Leroy, and V. Holloway Barbosa, "Skin from various ethnic origins and aging: an in vivo cross-sectional multimodality imaging study," Skin Research and Technology 15, 306-313 (2009).

[17] S. Lin, J. Wu, Ruei, H. Tan, W. Lo, W. Lin, T. Young, C. Hsu, J. Chen, S. Jee, and C. Dong, "Evaluating cutaneous photoaging by use of multiphoton fluorescence and second-harmonic generation microscopy," Optics letters 30, 2275-2277 (2005). PMID: 16190442.

[18] M. J. Koehler, A. Preller, N. Kindler, P. Elsner, K. König, R. Bückle, and M. Kaatz, "Intrinsic, solar and sunbed-induced skin aging measured in vivo by multiphoton laser tomography and biophysical methods," Skin research and technology: official journal of International Society for Bioengineering and the Skin (ISBS) [and] International Society for Digital Imaging of Skin (ISDIS) [and] International Society for Skin Imaging (ISSI) 15, 357-363 (2009). PMID: 19624433.

[19] M. Kaatz, A. Sturm, P. Elsner, K. König, R. Bückle, and M. J. Koehler, "Depth-resolved measurement of the dermal matrix composition by multiphoton laser tomography," Skin Research and Technology 16, 131-136 (2010). 